\title{
URGENCES PÉDOPSYCHIATRIQUES : RESITUER LES CRISES DANS LE CONTEXTE ORGANISATIONNEL DE LEUR SURVENUE
}

François Sicot

ERES | Empan »

2009/3 n 75 | pages 53 à 60

ISSN 1152-3336

ISBN 9782749211558

Article disponible en ligne à l'adresse :

http://www.cairn.info/revue-empan-2009-3-page-53.htm

\section{Pour citer cet article :}

François Sicot, «Urgences pédopsychiatriques : resituer les crises dans le contexte organisationnel de leur survenue », Empan 2009/3 (n 75), p. 53-60.

DOI 10.3917/empa.075.0053

Distribution électronique Cairn.info pour ERES.

(C) ERES. Tous droits réservés pour tous pays.

La reproduction ou représentation de cet article, notamment par photocopie, n'est autorisée que dans les limites des conditions générales d'utilisation du site ou, le cas échéant, des conditions générales de la licence souscrite par votre établissement. Toute autre reproduction ou représentation, en tout ou partie, sous quelque forme et de quelque manière que ce soit, est interdite sauf accord préalable et écrit de l'éditeur, en dehors des cas prévus par la législation en vigueur en France. Il est précisé que son stockage dans une base de données est également interdit. 


\section{DES \\ MUTATIONS \\ EN COURS...}

\section{pédopsychiatriques : \\ resituer les crises (1)}

dans le contexte

organisationnel

organisationnel
de leur survenue

François Sicot
Au départ des analyses qui suivent, le constat établi de manière empirique par des pédopsychiatres d'un grand nombre de services en France ${ }^{1}$ d'une augmentation des demandes d'avis psychiatriques, d'orientations et de prise en charge en urgence pour des adolescents en situation de rupture, «à risque », ou pour une urgence ressentie.

Par ailleurs, un certain nombre de publications et de polémiques récentes suggèrent que les enfants et adolescents ayant vécu des situations de rupture et de placement seraient plus exposés à présenter des troubles des conduites et un recours à la psychiatrie. Selon les estimations de ces pédopsychiatres, la proportion d'adolescents accueillis en urgence et qui présenteraient dans leurs antécédents des suivis ou placements ASE (Aide sociale à l'enfance) ou PJJ (Protection judiciaire de la jeunesse) pourrait atteindre entre 15 et $30 \%$. Comment expliquer l'accroissement de ces demandes d'accueil en urgence ? On présentera ici des éléments d'analyse issus d'une recherche en cours à partir d'un service d'urgences pédopsychiatriques $^{2}$. S'agissant des jeunes relevant d'une prise en charge judiciaire, sociale ou médico-sociale, on se demandera en particulier dans quelle mesure on peut dire que la prise en charge psy(chiatrique) relève de la mise en réseau et le recours aux urgences d'un défaut de partenariat ou de coordination.
François Sicot, professeur de sociologie, laboratoire LISST-CERS, université Toulouse 2-Le Mirail, 5 allées A.-Machado, 31058 Toulouse Cedex 9. sicot@univ-tlse2.fr

1. Médecins membres de la Société française de psychiatrie de l'enfant et de l'adolescent et des disciplines associées (SFPEADA) et de l'Association des psychiatres d'intersecteurs.

2. L'Équipe mobile de psychiatrie de l'enfant et de l'adolescent (EMPEA) n'est pas une équipe psychiatrique des Urgences mais une équipe mobile au sein d'un hôpital des enfants. Elle assure les urgences pédopsychiatriques, des consultations, la psychiatrie de liaison.

Je tiens à remercier chaleureusement l'équipe pour son accueil et son soutien, particulièrement M. Vignes et J.-P. Raynaud. 
3. I. Claudet, PH, M. Huguenin et C. Sérignac, assistantes sociales.

4. Cour des comptes, 2007.

5. " Le succès des services d'urgence tient aussi peut-être à des considérations économiques : s'il n'y a pas d'hospitalisation, le prix d'une simple consultation aux urgences dans un hôpital public n'est pas très élevé. II y a des possibilités de tiers payant et le paiement de la facture peut être longtemps différé ", Vassy, 2004, p. 67.

6. DREES, 2004

7. Cette augmentation des prises en charge ne correspond pas à une médicalisation des inadaptations ou déviances juvéniles car on la retrouve dans le social, le médico-social, le judiciaire. II s'agit donc plus exactement d'un phénomène d'accroissement généralisé de I'encadrement, Sicot, 2009.

8. De même que pour ce qui concerne les urgences, les statistiques disponibles ne nous informent que sur les services d'urgence proprement dits, enregistrés comme tels par la tutelle. Or, accueillir en urgence signifie bien plus largement accueillir le plus rapidement possible, pour une prise en charge non programmée, dans des services ou institutions très divers : CMP, CMPP, antennes mobiles...

9. "Dans la première moitié du $x x^{e}$ siècle encore, l'éducation des enfants était fondée sur les préceptes donnés par les parents et les exemples provenant de l'environnement familial.

\section{UN PHÉNOMÈNE SOCIAL NORMAL}

Dans le service concerné par l'enquête, les chiffres produits ${ }^{3}$ montrent une augmentation régulière et importante des admissions d'adolescents pour des troubles psycho-comportementaux sur la période 2001-2007, de 147 à 405 admissions, avec un pic à 495 en 2006. 13,5\% de ces adolescents provenaient d'un foyer ou d'un ITEP.

Il convient dans un premier temps de ne pas isoler cette tendance d'autres évolutions sociales au sein desquelles elle se situe. D'abord, on le sait, un recours croissant aux services d'urgences hospitaliers en général, avec un nombre de passages qui a doublé entre 1990 et 2004, de 7 à 14 millions ${ }^{4}$. Les explications sont nombreuses et connues, on se contentera de les rappeler : disponibilité d'un plateau technique polyvalent, accessibilité, fonction dispensaire qui n'est plus assurée par les structures extra-hospitalières, usage des urgences comme services de consultations non programmées, évolution de l'organisation des soins entre médecine de ville et hôpital, avec en particulier le retrait relatif de la médecine de ville de certaines de ses activités traditionnelles, acceptation de tout patient indépendamment de sa couverture sociale ${ }^{5}$.

D'autre part, ce sont les recours à la psychiatrie, publique comme privée, qui ne cessent d'augmenter depuis la fin des années 1980. Côté public, « le taux de recours aux secteurs de psychiatrie générale, qui était de $17 \%$ habitants en 1989 , atteint $26 \%$ habitants de plus de 20 ans en $2000^{6} \gg$.

Le nombre d'enfants et d'adolescents suivis par les secteurs de psychiatrie infanto-juvénile est, quant à lui, également en augmentation régulière et a doublé entre 1986 et $2000^{7}$. Un phénomène qui ne peut être expliqué par l'accroissement de la population juvénile en général puisque le «taux de recours » qui est «le rapport du nombre de patients suivis dans l'année par le secteur à sa population » (ici de moins de 20 ans) augmente lui-même pour passer de $16 \%$ en 1991 à $28 \%$ en 2000 . On remarquera en outre que la tranche d'âge des adolescents âgés de 15 ans et plus, qui était plutôt sous-représentée au début des années 1990, voit son taux de recours augmenter le plus rapidement entre 1991 et 2000. Enfin, la file active se renouvelle profondément : «Près de la moitié des enfants consultaient pour la première fois en 2000. »

Ces données ne nous renseignent pourtant que très partiellement sur l'évolution des prises en charge «psy » pour les jeunes ${ }^{8}$. En effet, il manque les données relatives à la pédopsychiatrie privée non sectorisée, au travail des psychologues et autres «intervenants psy » dont le nombre et la diversité sont difficilement évaluables. D'autre part, on sait qu'une part importante des prises en charge de difficultés «psy » sont réalisées par les médecins généralistes et que les prises en charge de ce type de problèmes ne relèvent pas 
seulement, pour les jeunes, de la psychiatrie, mais également des secteurs sanitaire, médicosocial, scolaire.

Le recours croissant à la psychiatrie pourrait s'expliquer par le décloisonnement de cette spécialité, sinon un affaiblissement de la stigmatisation qu'elle induit, le débordement de la pathologie par le domaine indéfini de la santé mentale, une détérioration objective de la santé mentale des populations. Du côté des jeunes, les mêmes explications sont avancées, oscillant entre emprise croissante de la souffrance (rapport HSCP), médicalisation du mal-être, des inadaptations et des déviances juvéniles, fragilisation des familles ${ }^{9}$.

\section{LE SERVICE D'URGENCE DANS SON CONTEXTE D'OFFRE DE PRISE EN CHARGE}

À étudier la littérature sur les urgences psychiatriques, il est notable que s'ignorent les approches macrosociales qui viennent d'être évoquées et des approches individualistes portant sur la clinique, l'étiologie des crises, les critères de distinction des vraies et des fausses urgences. Or, les premiers éléments d'une recherche en cours nous amènent à lier les deux approches : à restituer la crise dans l'environnement au sein duquel elle s'est déroulée. Deux niveaux de l'environnement doivent ainsi être pris en compte : celui de l'offre locale de prise en charge et celui de l'organisation des structures d'amont scolaires, sociales, médico-sociales - qui peuvent être amenées à recourir aux urgences.

Les flux de population d'un service d'urgences ne sont compréhensibles qu'à l'intérieur du système local de prise en charge auquel il appartient. Pour le service dont il est question ici, ce système est constitué d'une part d'une offre de prise en charge médicale générale et spécialisée. Sur le premier plan, il existe un dispositif dit de Permanence des soins (PDS) créé au niveau national en 2003. Dans ce cadre, il est demandé aux médecins généralistes d'assurer la permanence des soins supposée défaillante, ce qui, dans la rhétorique de la Cour des comptes, recouvre «la difficile articulation entre la médecine de ville et l'hôpital ». Avec cette limite que la participation des médecins libéraux aux gardes est volontaire. D'où des variations locales considérables dans la mise en place de la PDS. «C'est basé sur le volontariat. À Toulouse ville, par exemple, explique un interlocuteur, il n'y a pas de PDS parce qu'il n'y a pas de volontaires, ou très peu, donc il n'y a pas toujours de garde. Elle est mieux assurée en périphérie, mais avec des secteurs plus ou moins bien organisés. »

Or « l'analyse des passages horaires sur trois ans (2004-2006) dans le Pôle d'orientation spécialisé d'urgence (POSU) pédiatrique [de l'Hôpital des enfants de Toulouse] mettait en évidence une grande majorité (70\%) de recours après 18 heures ou pendant le week-end et les jours fériés. Cela tend à confirmer une demande en période de moindre permanence de soins ou d'insuffisance de consultations libérales non programmées » (Claudet, 2008).

Sur le plan des partenaires de la prise en charge psy des adolescents, on trouve, à côté des pédopsychiatres libéraux et des CMP - tous plus ou moins surchargés et ayant une file d'attente -, quatre structures qui accueillent les adolescents de moins de 16 ans en hospitalisation temps plein pour des périodes plus ou moins longues : une UCHA (Unité de crise et d'hospitalisation pour adolescents), une clinique privée, un hôpital de semaine en CHS pour les très jeunes et un service de CHU. «On a une palette, par exemple, quand on reçoit une TS (tentative de suicide), si c'est le début d'une pathologie très sévère et très compliquée ou qu'on pense qu'il va y avoir un placement ensuite, on s'achemine vers du long terme, on demande à ce qu'il soit pris en charge le plus tôt possible en hospitalisation. $\mathrm{Ou}$ bien on dit, c'est une crise très sévère, il va falloir organiser des synthèses avec les uns et les autres et puis il faut le tirer vers les grands, utiliser des contrats de soin: on oriente vers l'UCHA. Ou encore, si on est en situation de fragilité narcissique, on doit et on peut régresser un petit peu et c'est l'Hôpital des enfants. Donc, on a une palette. C'est pas : systématiquement cinq jours d'hospitalisation comme le recommande l'HAS. On tient compte de plusieurs para- 


\section{.../...}

La mère, dans l'immense majorité des familles, était constamment présente auprès de ses enfants qui étaient élevés dans une famille biparentale. La situation est, aujourd'hui, beaucoup plus complexe ; les structures familiales se sont affaiblies, soit que les deux parents travaillent et sont accaparés par leur activité professionnelle, soit que les enfants vivent dans des foyers recomposés ou monoparentaux où il n'y a plus de source incontestée de règles de vie... ", Académie nationale de médecine, 2003. Dans une autre perspective, Jamoulle, 2005. mètres, si des lits sont disponibles et qu'on a le choix, ce qui est rare » (EMPEA).

Plusieurs caractéristiques fondamentales de ces partenaires doivent être notées car elles expliquent la relation de service et de dépendance dans laquelle se trouve le service d'urgences par rapport à eux. À l'inverse de leurs partenaires, les établissements qui assurent le service public hospitalier ont une obligation de service public d'accueil de jour et de nuit. Les partenaires de l'EMPEA ont en effet des projets, n'accueillent les adolescents qu'après avoir évalué la pertinence ou l'adéquation de la demande à ce projet; ils ciblent des classes d'âge, voire des pathologies. L'un privilégiera les longs séjours, l'autre n'admettra qu'une semaine renouvelable une fois, le troisième sera fermé le week-end. Une structure n'équivaut donc pas à une autre, et l'on n'adresse pas un jeune vers l'une quand l'autre manque de place. «Ils ont chacun leur fonctionnement. Quand on appelle l'UCHA, ils nous disent, par exemple, j'ai une place, mais je ne peux pas parce que je n'ai pas une chambre sécurisée. Ou bien ils me disent, non cette ado, elle est très compliquée, le groupe est infernal, je le sens pas. On peut comprendre ça, il ne s'agit pas de démolir leur travail » (EMPEA).

Ces partenaires ont tous leur file d'attente, c'est-à-dire qu'ils refusent des jeunes faute de place, bien que leur profil corresponde au projet thérapeutique. Pour partie, l'engorgement résulte d'un défaut de possibilités de sortie pertinentes, soit vers le sanitaire (ambulatoire), soit vers le social ou le médico-social.

Les répercussions du manque de places adaptées se situent au sein des structures. Selon le degré d'autonomie dont il dispose, chacun des partenaires va devoir transiger plus ou moins avec son projet, sa population cible, augmenter sa durée de séjour.

« Vous avez évoqué une parenthèse entre votre prise en charge et les projets que vous mettez en place pour la sortie, elle est fréquente ?

- Oui, presque de manière systématique. On assure la transition mais sans en avoir les moyens, ce n'était pas le projet au départ. À un moment, une de nos psychologues était spécialisée, entre guillemets, dans cette transition. Elle recevait plus particulièrement les ados qui venaient de sortir. Mais elle n'est plus là et on n'a pas poursuivi » (UCHA).

L'EMPEA, quant à lui, ne disposant pas de lits de psychiatrie dédiés au sein de l'Hôpital des enfants, recourt à des lits de pédiatrie. D'autres services doivent à leur tour trouver une solution pour leurs patients, en les adressant parfois à d'autres hôpitaux.

Mais par ailleurs, ces répercussions sont systémiques : les difficultés de l'un ont immédiatement des retombées sur les autres. Les parcours de prise en charge des jeunes sont étroitement liés à un système de prise en charge local. La crise de l'adolescent s'inscrit 
dans un contexte où les professionnels et les structures ont à gérer leur propre manque de moyens, leur débordement face à la demande et celui des autres. Le fait d'être admis aux urgences en pédopsychiatrie n'est finalement qu'un cas particulier de ce phénomène plus global de parcours contraint.

Ce système local d'offre sanitaire, outre qu'il doit répondre aux demandes directes des familles des jeunes, des médecins libéraux, des lieux de soins psychiatriques ambulatoires, est imbriqué dans un système plus large de prise en charge des souffrances et inadaptations juvéniles. L'EMPEA, comme les quatre autres services d'hospitalisation pédopsychiatrique, reçoit des demandes émanant des secteurs social, médico-social et judiciaire tels que MECS, ITEP ou du Centre départemental de l'enfance et de la famille (CDEF).

Le CDEF, par exemple, accueille une population cumulant les difficultés sociales, «psy» et fréquemment judiciaires. La violence n'est pas rare et les besoins de prise en charge psychiatrique sont nombreux. À l'instar de l'EMPEA, le CDEF a pour caractéristique une obligation d'accueil : « Ici, on est un centre de protection de l'enfance, on reçoit avant tout les enfants victimes. Pour moi, c'est incompatible de mettre dans le même espace des enfants victimes de sévices sexuels et les agresseurs. Et pourtant, on nous impose ça, on n'a pas le choix. Ils viennent en OPU [Ordonnance de placement en urgence] et on est obligés de les prendre. C'est une décision de justice, le gamin, on ne peut pas le laisser dans la rue [...]. On est le seul établissement d'urgence à être ouvert 24 heures sur 24. Comme il y a très peu de lits psychiatriques, on nous impose aussi ces situations-là. »

Les difficultés, pour les professionnels des secteurs social et médico-social, pour faire hospitaliser un jeune en psychiatrie sont bien connues: réticence des parents, de la tutelle, intrication des difficultés sociales, judiciaires et donc de la préséance du symptôme. Un des partenaires remarque ainsi : «Ces jeunes sont dans des situations de rupture sociale majeure, ils sont déjà parfois largement dans la délinquance et malgré tout, malgré cette délinquance, ils présentent des troubles psychiatriques. C'est pas du tout incompatible d'être dans la délinquance et d'avoir des troubles psychiatriques.

- Vous faites appel aux urgences?

- Oui, on est bien obligés. Par incapacité ici, dans des crises, à les gérer. Le dernier cas, c'était la semaine dernière, Michel. Il a frappé sur sa sœur, il l'a vraiment amochée. Il est ici en ce moment. Enfin, s'il n'a pas refugué. Parce que comme il devait être hospitalisé à la clinique $\mathrm{X}$ et que la clinique a pensé qu'ils ne pouvaient pas le contenir, ils n'ont pas pu l'admettre. Alors que ça fait une semaine qu'on cherche un lit pour lui, c'est pour lui que j'ai téléphoné au CHS. »

Une telle manière de situer un service d'urgence au sein d'un système local de prise en charge a peu à voir avec la promotion, par les politiques publiques, de la collaboration, du partenariat, de la mise en réseau. Le problème paraît en effet moins celui d'une collaboration insuffisante que celui des moyens dont se dotent toute organisation, toute structure pour maîtriser leur activité et des ressources dont elles disposent pour mener à bien leur action.

\section{LA CRISE ET SA SURVENUE DANS LES STRUCTURES SOCIALES ET MÉDICO-SOCIALES}

Une partie des recours aux urgences émane d'institutions sociales, médico-sociales et, plus rarement, judiciaires; elle concerne donc des jeunes qui font, par ailleurs, l'objet d'une prise en charge sociale, médico-sociale ou judiciaire.

«L'urgence est classiquement considérée comme l'évolution d'une crise méconnue, qui n'aurait pas eu les réponses appropriées » (Guedj et Caroli, 1997). «L'entourage ou le jeune vont rapidement mettre en exergue ces symptômes, les amplifier et les médicaliser pour s'en débarrasser au plus vite et ne pas remettre en question l'équilibre du système » (Laudrin et Speranza, 2002).

Éric, 15 ans, est hébergé en MECS alors qu'une orientation MDPH (Maison départementale des 
10. $12 \%$ des admissions ont eu lieu après minuit en 2007 contre 7 à $9 \%$ dans la période précédente.

11. On notera que les foyers de la Protection judiciaire de la jeunesse bénéficient, sur Toulouse, d'un encadrement éducatif conséquent la nuit.

12. Temporalités des symptômes, des organisations, du politique, pas toujours conciliables: "En psychiatrie, on a une DAF [Dotation annuelle de fonctionnement ] qu'ils nous donnent en début d'année, en diminution tous les ans, autour de 2 ou $3 \%$ de moins, que nous donne I'ARH, c'est-à-dire la tutelle. On nous donne tant de DAF et avec ça on doit fonctionner, c'est-àdire tout payer : le personnel, les médicaments et tout le fonctionnement.

- L'augmentation des jeunes en urgence, I'ARH elle est au courant de ça?

- Oui bien sûr, les médecins le disent, c'est publié. La réponse, c'est de faire des projets, des projets ciblés qui sont financés. Par exemple dans le cadre du plan de santé mentale. II faut que ça passe par le SROS, il faut que ce soit agréé et à ce moment-là, on nous donne le financement pour ces projets. Mais souvent ces projets ne financent que du personnel, c'est là où ça nous coince. Le fonctionnement quotidien, il faut qu'on le prenne dans la DAF. Et puis c'est long. Le petit hôpital de jour ça doit faire trois ans qu'on en parle " (gestionnaire au $\mathrm{CHU}$ dont dépend I'EMPEA). personnes handicapées) en ITEP avait été notifiée mais n'a pu se réaliser, faute de place. Depuis plusieurs semaines, ses parents, qui devaient le reprendre au domicile, repoussent leur décision. « $\mathrm{Au}$ départ, explique l'éducateur, c'était posé comme provisoire avec lui et avec nous, et malheureusement, ça a perduré. » Le comportement d'Éric s'aggrave : « Il fallait trouver des temps plus individuels pour le protéger et protéger les autres et lui permettre de souffler un peu. Il était demandeur. Beaucoup d'angoisses le soir, d'angoisses nocturnes... Il s'enfermait dans une relation exclusive et quand on ne répondait pas aux demandes, il avait des crises. Des crises importantes et ça faisait peur aux autres enfants. Il balançait tout dans sa chambre, il provoquait les autres, il les insultait. » L'équipe de la MECS finit par envisager, en synthèse, une prise en charge par l'UCHA et celle-ci est mise en attente lorsque survient une crise plus violente que les autres : «Le vendredi soir, quand on lui expliqué qu'il n'irait pas en week-end avec son père mais qu'il irait à l'UCHA, il a commencé à s'agiter, il a fallu le faire sortir du bureau, l'aider à se calmer, le contenir... » L'hospitalisation à l'UCHA se réalise donc sur une première crise. Éric est ensuite placé en famille d'accueil spécialisée. Une semaine seulement après cette orientation, il est admis aux urgences. Le rapport de transmission du service de nuit contient les notes suivantes : «Trouble du comportement ce soir dans sa famille d'accueil. Attouchements sexuels envers les enfants de la famille. Ce soir, agressivité et recadrement par les parents d'accueil. » Le travail de l'EMPEA consistera, après les soins, à trouver une solution de sortie.

Un praticien hospitalier de l'Hôpital des enfants déclare, à propos des foyers, des structures sociales ou médico-sociales : «Ce sont des structures qui devraient être un peu plus contenantes, ce sont des professionnels, qui nous adressent les jeunes comme ça, en ambulance, pas forcément accompagnés. Parce qu'il a molesté un éducateur, on envoie un jeune dans une structure d'urgence pédiatrique, qui n'est pas formée à ça, au milieu de jeunes enfants. »

Robert, 14 ans et demi, arrive aux urgences, le 11 octobre 2008, vers minuit, amené par des policiers et accompagné d'un éducateur de l'iteP dans lequel il réside. Le médecin de garde a noté : «Entré pour un état d'agitation clastique dans la nuit à la suite d'une frustration. Une éducatrice se serait opposée à lui à la suite d'une tentative de cadrage. »

Avec Robert, depuis deux ans en ITEP, des difficultés apparaissent tôt dans la prise en charge : il ne s'implique pas dans les activités, ne fait qu'y passer, «n'accroche » à aucune des activités proposées, «ne tient pas en place ». Dès la première année également, « il est amené à suivre les jeunes les plus en difficulté, à faire des bêtises. Sans qu'il en ait vraiment conscience et sans qu'on puisse avoir vraiment de prise là-dessus » (éducatrice). Les derniers mois qui précèdent l'entrée aux urgences sont décrits sur le mode de la 
montée en puissance des comportements conflictuels et des difficultés grandissantes de l'équipe à les apaiser ou à les contrôler. Face à cette dégradation, l'équipe de l'ITEP envisage une possible réorientation, en se posant beaucoup de questions sur ce qui aurait pu lui convenir. Un établissement médico-social type IME est discuté du fait de ses limites intellectuelles, certains membres de l'équipe évoquant l'intérêt d'une hospitalisation en pédopsychiatrie aux fins de diagnostic, "pour approfondir les choses ». La crise qui amène Robert aux urgences résulte, quant aux événements, d'un refus d'aller se coucher, d'une tentative de l'éducatrice, qui fait appel au veilleur de nuit, de faire rentrer Robert de force, d'un appel du SAMU par les deux professionnels qui n'arrivent pas à le raisonner, à stopper les dégradations, à le faire sortir de sa chambre et, finalement, devant l'échec du SAMU, du recours à la police. « Le SAMU n'est pas arrivé à l'attraper. Donc, ils ont appelé la police. La police est arrivée, je ne sais pas comment ça c'est passé, il est parti menotté, jusqu'aux urgences. »

Si aux urgences pédiatriques de Toulouse les deux tiers des admissions des adolescents pour troubles psychiques se font sur les horaires de garde ${ }^{10}$ ou les jours fériés, le phénomène ne s'explique pas seulement par le défaut de permanence des soins mais également par l'organisation du travail au sein des structures sociales ou médico-sociales ${ }^{11}$.

On retrouve ici, comme dans un autre cas, le problème auquel sont confrontées les structures sociales et médico-sociales en fin de soirée et qui expliquerait pour partie l'importance du nombre d'adolescents accueillis aux urgences en fin de soirée ou de nuit, en provenance des institutions : un encadrement éducatif réduit au strict minimum puis la présence du seul veilleur de nuit. « À partir de 23 heures, il n'y a que le veilleur de nuit. Moi, j'y mettrai pas mon enfant. Je le dis. C'est vrai que c'est problématique. Avant, les éducateurs faisaient les nuits » (éducatrice).

Les données du service d'urgence pédiatrique montrent qu'en 2007, 44 adolescents cumulaient 117 passages, soit en moyenne trois admissions en urgence dans l'année. Comment expliquer cette réitération ? Pour le médecin de l'Hôpital des enfants interviewé : «C'est sans doute que l'endroit où ils sont n'est pas approprié, soit que la structure qui les héberge ne trouve pas elle-même de réponse à l'état de ce jeune et donc utilise les urgences comme un tremplin ou une plate-forme pour qu'il soit réorienté vers une autre structure. Ça n'est sans doute pas le rôle du CHU et des urgences. »

Marine, 14 ans, est une de ces adolescentes. Elle est hospitalisée une première fois six jours aux urgences suite à des crises clastiques au domicile, des insultes envers les parents, des idéations suicidaires. La sortie s'effectue vers l'UCHA qui, après deux semaines d'hospitalisation, l'adresse au CMPP de son secteur. Celui-ci ne pouvant l'accueillir, c'est l'UCHA qui met en place des visites à domicile - activité qui ne relève pourtant pas de son projet. Très rapidement, elle est de retour aux urgences suite à des crises d'angoisse : «La famille a fait venir le médecin traitant qui les a orientés aux urgences »(dossier). Une nouvelle prise en charge par l'UCHA est envisagée mais cette fois, Marine la refuse. Le suivi CMPP se met en place. Six mois plus tard, nouvelle admission aux urgences pour «pour crise d'agitation clastique au domicile ». La sortie se fait à nouveau vers l'UCHA, un médecin de l'EMPEA notant: «Cette nouvelle hospitalisation intervient, d'une certaine façon, par défaut de possibilité d'accueil rapide à l'hôpital de jour. Cette hospitalisation a été cette fois acceptée par les deux parents et Marine elle-même. Nous avons proposé un séjour intermédiaire à l'UCHA dans la mesure où, au printemps dernier, la mise en place de soins adaptés s'était heurtée à de nombreuses difficultés et à des résistances considérables. »

Le cas de Marine illustre la complexité des réitérations, qui résultent ici à la fois d'une absence de disponibilité des structures mais aussi des réticences de l'adolescent et/ou de ses parents, sans oublier le temps de la pathologie ${ }^{12}$.

\section{Conclusion}

Le décalage est frappant entre, d'une part, la connaissance à la fois scientifique, empirique par 
les professionnels, politique de l'augmentation de la demande sociale de prise en charge «psy » des souffrances des adolescents et, d'autre part, l'insuffisance des moyens effectivement mis en œuvre dans les politiques publiques. Les institutions sanitaires, sociales et médicosociales sont de fait en situation de devoir « tenir ». Le partenariat, le travail en réseau, sans cesse promus et valorisés, peuvent dès lors apparaître comme des moyens de gérer au moindre coût, par la mutualisation, le manque de ressources de chacun des partenaires. Dans cette perspective, «l'incasable», le jeune qui tourne d'une structure à l'autre, qui les use toutes ou « les fait toutes péter », est moins le produit de caractéristiques individuelles, intrinsèques, que celui d'une politique publique.

Plus encore que les autres services hospitaliers dont le recours dépend essentiellement des besoins de la population, les services d'urgence dépendent d'abord des moyens et de l'organisation du travail des partenaires.

\section{BiBLIOGRAPHIE}

ACADÉMIE NATIONALE DE MÉDECINE. 2003. La santé mentale de l'enfant de la maternelle à la fin de l'école élémentaire, rapport.

Baubeau, D. ; CarRasco, V. 2003. " Motifs et trajectoires de recours aux urgences hospitalières », Études et résultats, ${ }^{\circ} 215$, janvier, DREES.

Claudet, I. ; Joly-Pedespan, L. 2008. "Consultations de routine aux urgences: faut-il gérer ou lutter?", Archives de pédiatrie, $\mathrm{n}^{\circ} 15$, p. 1733-1738.

COLDEFY, M. ; Salines, E. 2004. « Les secteurs de psychiatrie générale en 2000 : évolutions et disparités », Études et résultats, n 342 , octobre, DREES.

COUR DES COMPTES. 2007. Les urgences médicales : constats et évolution récente, rapport.

GuEDJ, M.J. ; CAROLI, F. 1997. « Accueil et soins différentiels pour adolescents : urgence et hospitalisation aiguë », Psychiatrie de l'enfant, XI, 1, p. $239-272$.

HAUT COMITÉ DE LA SANTÉ PUBLIQUe. 2000. La souffrance psychique des adolescents et des jeunes adultes, ministère de l'Emploi et de la Solidarité.

JAMOULle, P. 2005. «Fragilisation de la figure paternelle dans les cités sociales », dans J. Furtos et Christian Laval (sous la direction de), $L a$ santé mentale en actes, Toulouse, érès/ORSPERE.

LAUdRIN, S. ; SPERANZA, M. 2002. « Crise et urgence en pédopsychiatrie », Enfances\&PSY, $\mathrm{n}^{\circ} 18$, p. 17-23.

RobIn, M. ; Pochard, F. et coll. 2001. «Les dispositifs d'urgence psychiatrique et de crise en France », Thérapie familiale, vol. 2, n 22, p. 133-151.

Sicot, F. 2009. « Une reconfiguration du traitement des déviances juvéniles au tournant des années 1990 : vers un accroissement généralisé de l'encadrement », dans J.-C. Caron, A. Stora-Lamarre (sous la direction de), Les âmes mal nées. Jeunesse et délinquance urbaine en France et en Europe (XIX'-XXI siècle), Presses universitaires de Franche-Comté.

VASSY, C. 2004. «L'organisation des services d'urgence, entre le social et le sanitaire », Mouvements, $\mathrm{n}^{\circ}$ 32, p. 67-74. 